\title{
Preganglionic Epidural Steroid Injection through Translateral Recess Approach
}

\author{
Seok Min Hwang, MD, In Seok Son, MD*, Pei Juin Yang, MD, Min Seok Kang, MD* \\ Department of Orthopedic Surgery, Seoul Red Cross Hospital, Seoul, \\ * Department of Orthopedic Surgery, Konkuk University Medical Center, Konkuk University School of Medicine, Seoul, Korea
}

The approach we suggest was developed for cases in which the fourth and fifth lumbar and first sacral spinal nerves were affected in lumbar degenerative disc disease. Retrodiscal transforaminal epidural injection is known to be very effective for lumbar radiculopathy because of excellent access to primary pathology; however, access below L5 is often restricted by the anatomic characteristics of the L5-S1. In the translateral recess approach (TLR), proper final needle placement (i.e., in the axillary portion between the exiting and traversing nerve roots) can be achieved by setting the direction of the needle laterally and superiorly from the distal tip of the infra-adjacent spinous process toward the medial wall of the pedicle and neural foramen of the given level without neural injury. This approach is possible because of the wide interlaminar space in the L5-S1. Preganglionic epidural injection through TLR is an effective and safe spinal intervention for lumbosacral radiculopathy.

Keywords: Preganglionic epidural injection, Translateral recess approach, Lumbosacral radiculopathy

Among various spinal interventions for back pain, epidural steroid injections are most widely used ${ }^{1)}$ and have sufficient therapeutic potency to provide a small surgerysparing effect in the short term and may reduce the need for surgery in some patients. ${ }^{2)}$ In particular, preganglionic epidural injections through the retrodiscal transforaminal approach may significantly improve clinical outcomes compared to those of any other epidural modalities in patients with lumbosacral radiculopathy regardless of age, sex, duration of symptoms, and pain intensity. ${ }^{3)}$ However, this approach may be restricted by the interference of the iliac crest and orientation of the facet at the lumbosacral level. ${ }^{4}$ Here, we suggest an alternative approach, which we named "translateral recess approach for preganglionic epidural injection" to address some of these drawbacks.

\footnotetext{
${ }^{*}$ Current affiliation: Seoul Chuk Hospital, Uijeongbu, Korea Received July 20, 2018; Accepted September 5, 2018 Correspondence to: Min Seok Kang, MD Seoul Chuk Hospital, 6 Taepyeong-ro, Uijeongbu 11698, Korea Tel: +82-2-1599-0033, Fax: +82-31-830-2264

E-mail: orthopedicspinae@gmail.com
}

\section{TECHNIQUE}

Preganglionic epidural injections through the translateral recess approach were performed on an outpatient basis by a single spine surgeon (MSK). Patients were placed prone on a radiology table. Patients' back was prepared by using an iodine-based antiseptic solution. After taking a standard anteroposterior (AP) view at the corresponding level under fluoroscopic guidance, a 20G 3.5-inch Tuohy needle was inserted into the distal tip of the distal adjacent spinous process (or tubercle) and advanced toward the medial wall of the proximal pedicle (Fig. 1). Next, after taking a standard lateral view, the needle was inserted into the corresponding neural foramen and adjusted as close and parallel to the proximal lamina as possible (Fig. 2). Then, 0.5 $\mathrm{mL}$ of contrast dye (Iobrix 300, Iohexol; Taejoon Pharm, Seoul, Korea) was injected through the needle tip placed in the dorsal epidural space below the ligamentum flavum to determine the epidurographic pattern. After confirming that the needle tip was placed outside the posterolateral margin of the thecal sac on the standard AP and lateral views, the needle was gently advanced until it touched the posterior aspect of the proximal vertebral body or the annulus fibrosus of the intervertebral disc. Contrast dye (1 

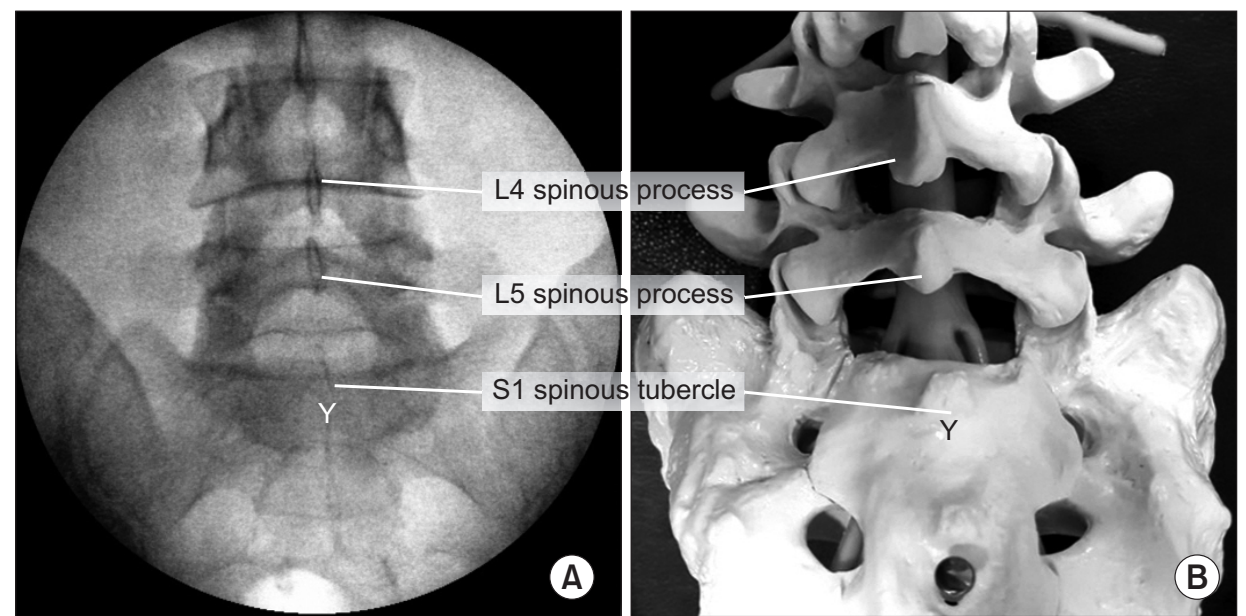

Fig. 1. Entry points of preganglionic epidural injection through translateral recess approach. (A) Distal portion of the S1 spinous tubercle has a " $Y$ " shape in the lumbosacral standard anteroposterior
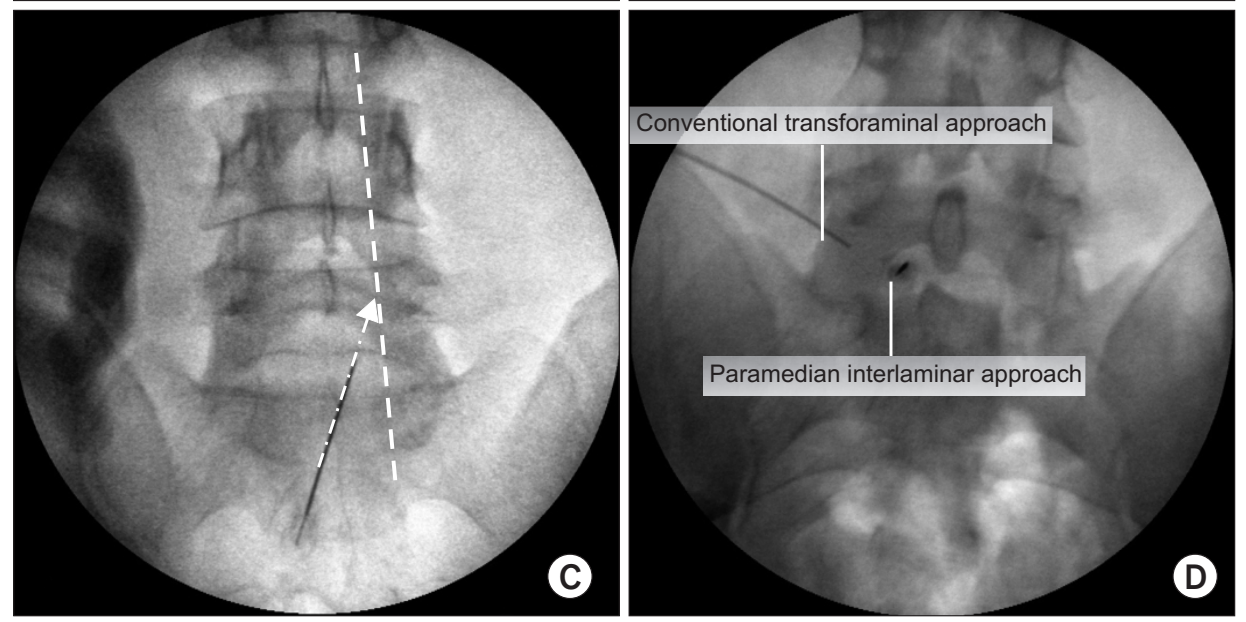
(AP) image. Under fluoroscopic guidance, a 20G 3.5-inch Tuohy needle was inserted into the distal tip of the S1 spinous tubercle. (B) AP image of an artificial lumbar spine model. (C) Under fluoroscopic guidance, a 20G 3.5-inch Tuohy needle was advanced toward the medial wall of the proximal pedicle. Dotted white line: medial pedicular line, Dotted white arrow: the direction of the Tuohy needle in the AP image. (D) Needle position of the conventional transforaminal approach and the paramedian interlaminar approach in the AP fluoroscopic image.

$\mathrm{mL}$ ) was then injected to determine the epidurographic pattern on the standard AP and lateral views. After the ventrolateral epidural space was confirmed, $1 \mathrm{~mL}$ of dexamethasone $(5 \mathrm{mg}$ ) with $2 \mathrm{~mL}$ of $0.2 \%$ ropivacaine and 4 $\mathrm{mL}$ of sterile normal saline were injected (Fig. 3). Patients were monitored for at least 30 minutes after the procedure and were discharged if they were able to walk without any complications.

\section{Case Illustration}

A 57-year-old man presented with axial back pain (visual analog scale [VAS], 5) and left leg pain (VAS, 7) which radiated to the posterior aspects of the thigh, calf, and foot plantar (S1 territory) for 2 months. He also had pain referred to the left posterior thigh and a gait disturbance with a pattern of neurogenic claudication. He had a history of left side discectomy performed at L4-5 25 years ago.

Neurological examination revealed negative straight leg raise on both sides and decreased motor strength (4/5) in plantar flexion of the left ankle. Anal sphincter tone was intact. Sensation over the left S1 nerve root dermatomes was diminished. Plain radiography showed lumbar spondylosis with discovertebral degeneration at L4-5. Magnetic resonance imaging showed the previous left partial hemilaminectomy of the L4 vertebra and left S1 nerve root compression due to left subarticular lumbar disc herniation at L5-S1. The patient provided a signed informed consent form before the epidural injection. He underwent a preganglionic epidural injection through the translateral recess approach (Fig. 4).

Two weeks after the injection, radicular leg pain completely disappeared, and axial back pain improved from 5 points to 2 points. There were no further changes in clinical manifestation until 8 weeks after the injection. Three months after the injection, the patient complained that referred pain around the left buttock slightly deteriorated.

\section{DISCUSSION}

Given the anatomic characteristics of the lumbosacral level, which has an interference of the iliac crest, a large facet 

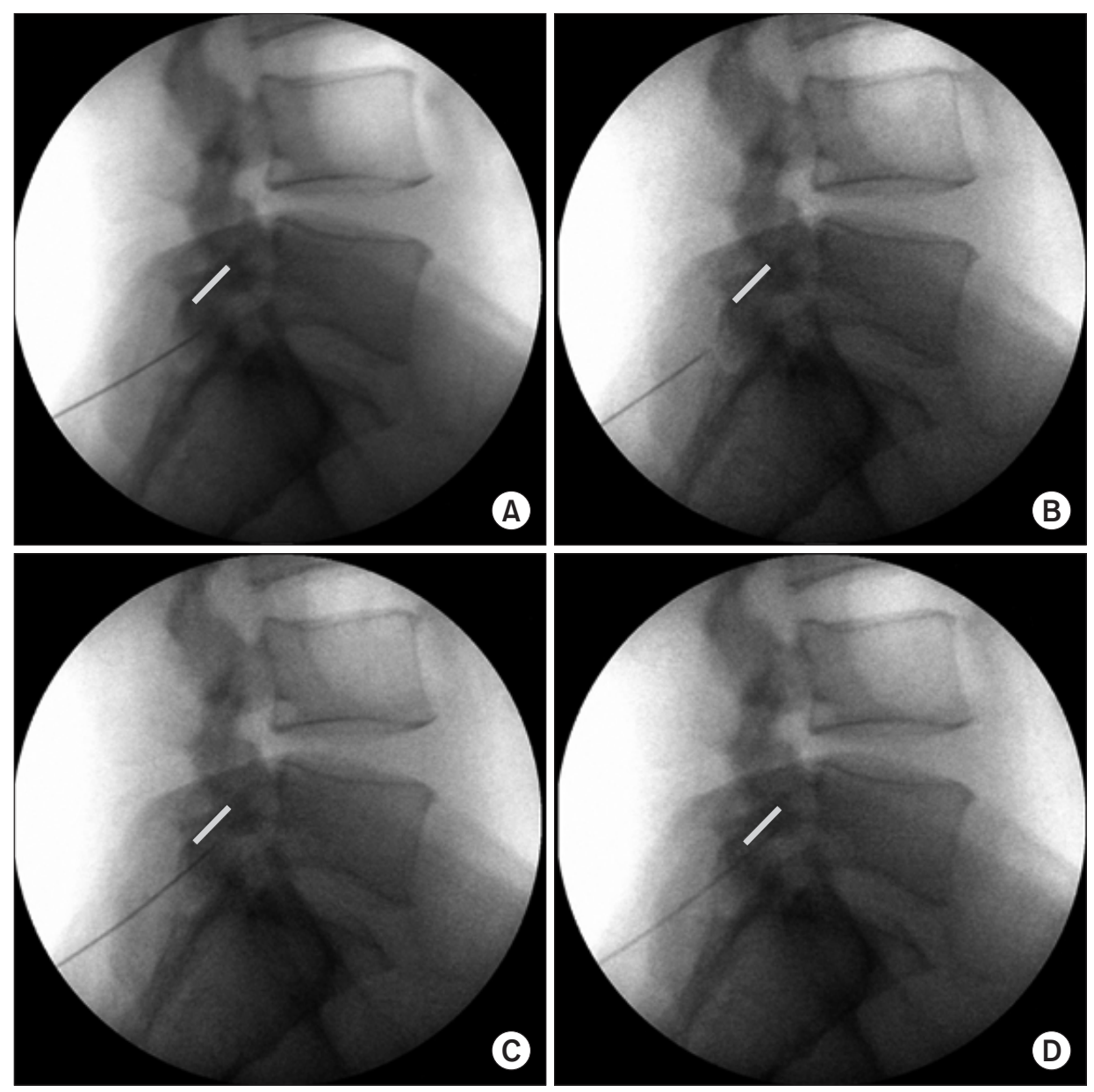

Fig. 2. Adjustment of the sagittal incidence angle of Tuohy needle. (A) Standard lateral image showing a Tuohy needle is stuck in the ligamentum flavum immediately after needle insertion. The Tuohy needle was pulled back (B) and its orientation was adjusted (C) to be inserted into the given neural foramen as close and parallel to the proximal lamina as possible. (D) Standard lateral image showing the Tuohy needle entering the dorsal epidural space through "loss of resistance technique." Bold gray line: undersurface of $L 5$ lamina.

joint, narrow foramen, and wide inter-laminar space, ${ }^{4)}$ we considered that epidural injection through the interlaminar space would be much more advantageous than the approach through the neural foramen. The interpedicular distance and interlaminar width increase from L3-4 to L5-S1. ${ }^{5)}$ The ratio of the transverse diameter of the dural sac to the interpedicular distance tends to be maintained at about $60 \%,{ }^{6}$ whereas the ratio of the transverse diameter of the dural sac to the interlaminar width tends to decrease from the L3-4 to L5-S1 level (82\% at L3-4, 65\% at L4-5, and $61 \%$ at L5-S1) (Table 1). These features suggest from the L3-4 to the L5-S1 level, the space outside the dural sac will be sufficiently wide to insert the Tuohy needle. In the standard paramedian interlaminar approach, after obtaining the most widely visible AP image of the interlaminar space of a target level under fluoroscopy, the needle is inserted into the center of the affected hemisphere of the interlaminar space in the AP plane and directed inward at $10^{\circ}$ to $15^{\circ}$. However, as suggested by the authors, if the needle direction is set laterally of the center of the interlaminar space to the medial wall of the pedicle, and the needle tip is located outside the dural sac when placed in the dorsal epidural space through the ligamentum flavum, the risk of intrameningeal injection due to dural puncture is expected to be minimized even if the needle is advanced to the ventral epidural space. In the sagittal plane of the lumbar spine, the laminar height was shorter at L5 than L4 (L4, $20.4 \pm 3.0 \mathrm{~mm}$; L5, $16.6 \pm 3.1 \mathrm{~mm}$ ), and the laminar slope angle was similar regardless of the spine level (L4, $117.5^{\circ} \pm 4.0^{\circ} ; \mathrm{L} 5,115.7^{\circ} \pm 5.7^{\circ}$ ) (Table 1 ). Considering the laminar overhanging and slope angle, ${ }^{7)}$ in order to position the epidural needle tip at a given neural foramen level, the entry point should be set below the given intervertebral disc, and the needle should enter the laminar as close and parallel as possible. Therefore, we propose to set the entry point at the distal tip of the infra-adjacent spinous process or tubercle.

Among various spinal interventions for lumbar degenerative disc disease, epidural injections are most widely used, with their use more than doubling in the last decade. ${ }^{1)}$ They have been reported to provide a small surgery-sparing effect in the short term and reduce the need 

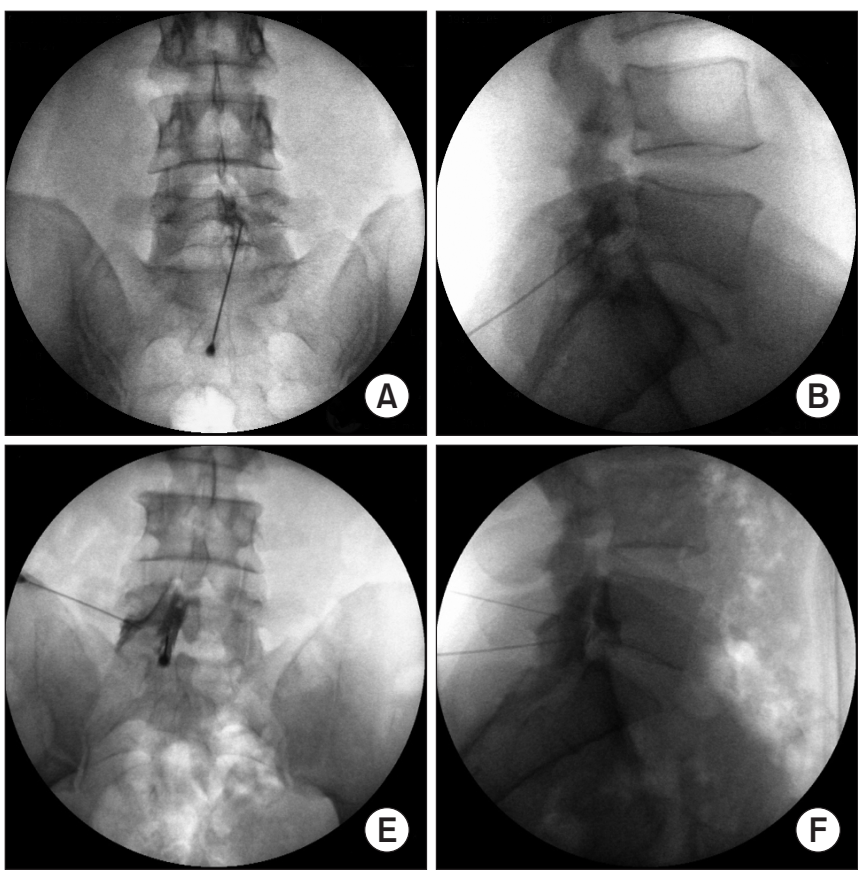
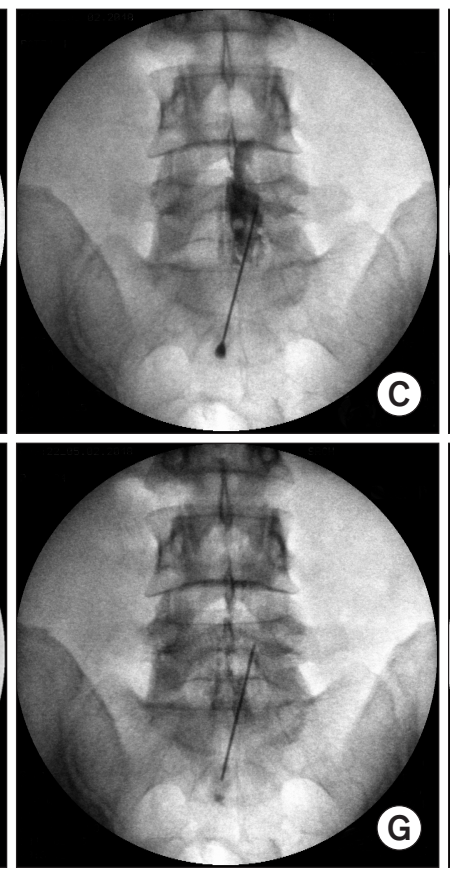
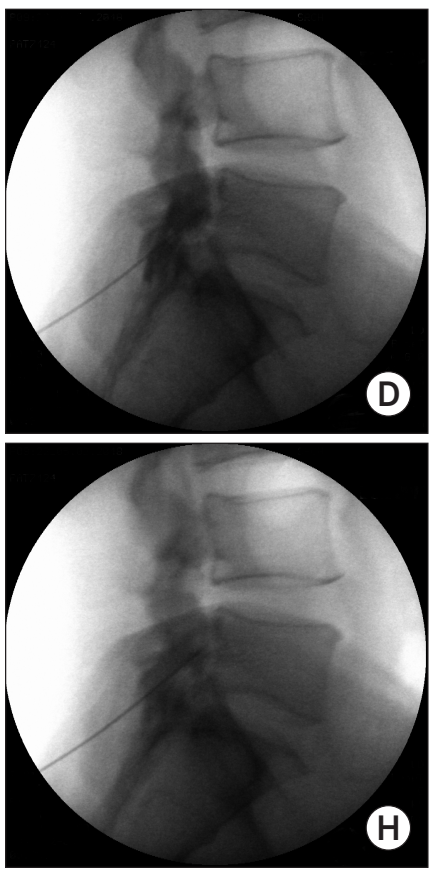

Fig. 3. Needle tip position and epidural contrast spread pattern. After placement of the Tuohy needle in the dorsal epidural space via the ligamentum flavum, $0.5 \mathrm{~mL}$ of contrast dye (lobrix 300, Iohexol; Taejoon Pharm, Seoul, Korea) was injected to determine the contrast dispersal pattern on the anteroposterior (AP; A) and lateral (B) fluoroscopic images. The position of the end of the needle was on the outside of the $L 5$ traversing nerve root in the coronal plane (A) and in the dorsal epidural space in the sagittal plane (B). Then, the needle was gently advanced until it touched the posterior aspect of the proximal vertebral body or the annulus fibrosus of the intervertebral disc. It was observed that the final needle tip position rose from the inferior pedicle line to the mid-height of the pedicle on the AP (C) and lateral (D) fluoroscopic images. It was also confirmed that the contrast medium spread out along the $L 5$ exiting nerve root and $S 1$ traversing nerve root (C), as well as into the ventral epidural space (D). However, in paramedian interlaminar epidurography, the needle tip was $10^{\circ}-15^{\circ}$ inward and located inside the lateral margin of dural sac (E), and dorsal epidural contrast spread (F) was observed. In conventional transforaminal epidurography, ventral epidural contrast spread (F) and periradicular infiltration (E) were observed along the L5 exiting nerve root. After drug injection, periradicular infiltration $(G)$ through the $L 5$ exiting and $S 1$ traversing nerve roots was noted and contrast dye was observed to spread from the ventral epidural space $(\mathrm{H})$ in the craniocaudal direction on the $\mathrm{AP}(\mathrm{G})$ and lateral $(H)$ fluoroscopic images.

for surgery in some patients. ${ }^{2)}$ The therapeutic potential of epidural injections is eventually determined by the approach to the main pathology and injectate composition. For this reason, preganglionic epidural injections through the retrodiscal transforaminal approach reportedly result in the most favorable clinical outcomes of any epidural modality in patients with lumbosacral radiculopathy regardless of age, sex, duration of symptoms, and pain intensity. ${ }^{3)}$ However, the applicability of the retrodiscal transforaminal approach may be limited due to the interference of the iliac crest and the orientation of the facet joint at the lumbosacral level as well as neural foraminal stenosis due to the hypertrophy of the superior articular process and loss of disc height. ${ }^{4)}$ Also, intradiscal injection-related complications occur at a relatively high frequency.

The method we suggest, named as the translateral recess approach, has been developed to be applied to below L4-5, which is difficult to access by the retrodiscal transforaminal approach. The most commonly affected site of lumbar spondylosis is the L4-5 level, followed by the L5-S1 and L3-4 levels. ${ }^{4)}$ The translateral recess approach suggested by the authors enables access to the lateral recesses at the L4-5 and L5-S1 levels. However, the anatomical characteristics such as the long laminar length, narrow interlaminar space, and wide neural foramen in the upper lumbar region do not allow the translateral recess approach; access to the preganglionic epidural space through the retrodiscal approach is rather easy. ${ }^{9)}$

In conclusion, the technique presented here is an effective and safe intervention, which can be used as an alternative approach for patients who have radiculopathy at or below L4 with lumbar degenerative disc disease. The important advantage of this technique is the accessibility of the ventral epidural space without interference of the dural sac. Also, an entry point is accessible on each side at the same level. 
Hwang et al. Translateral Recess Epidural Injection

Clinics in Orthopedic Surgery • Vol. 11, No. 1, 2019•www.ecios.org
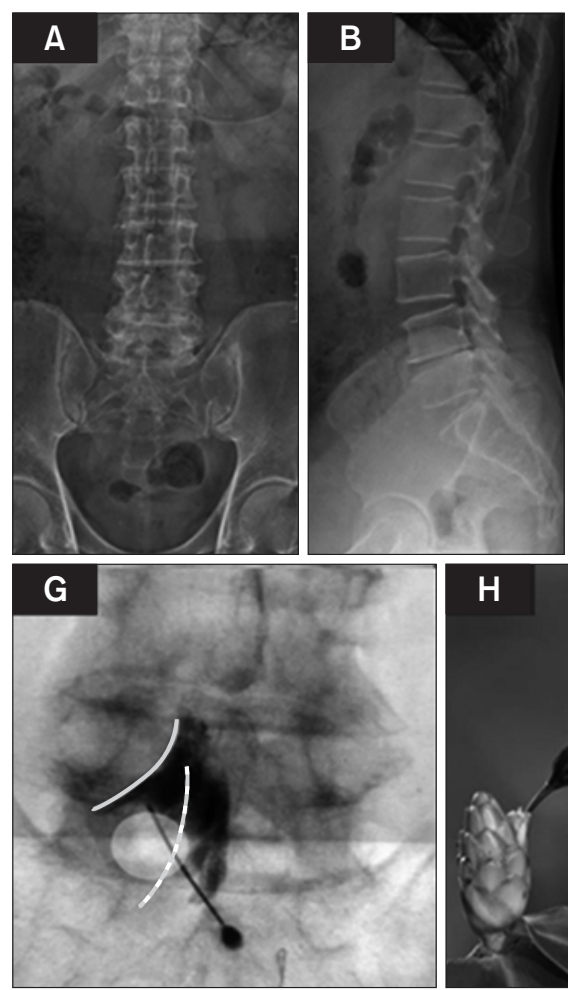
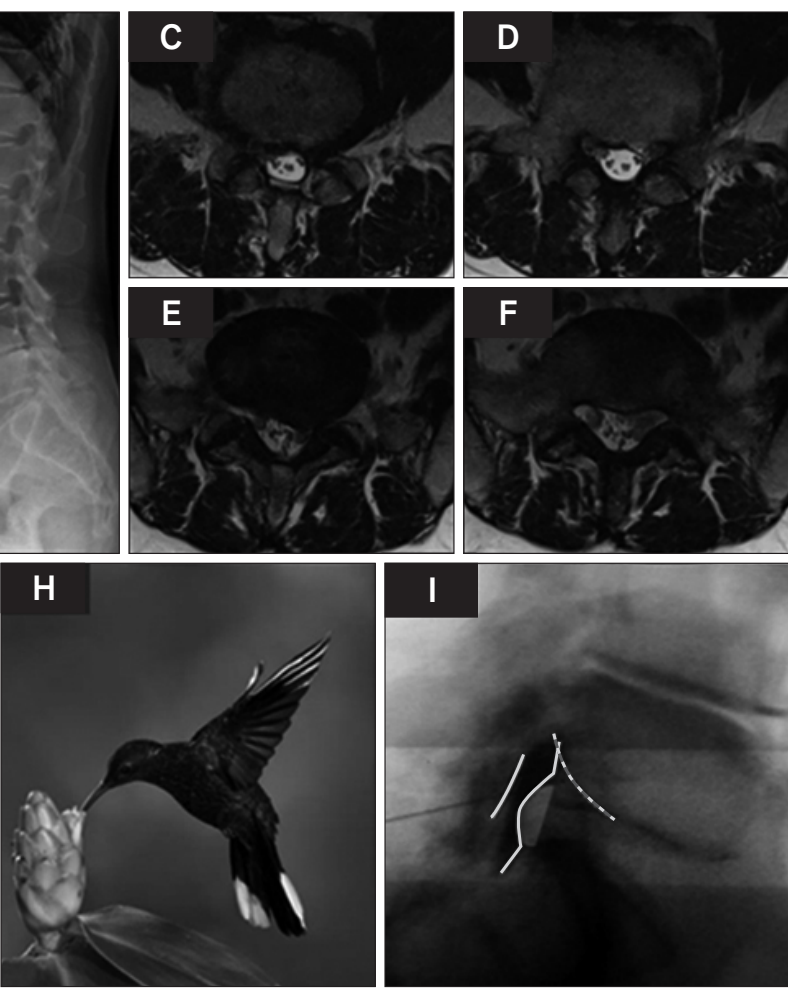

Fig. 4. Epidurography of a patient with subarticular disc extrusion and downward migration at L5-S1. The patient underwent a preganglionic epidural injection through the translateral recess approach. Plain anteroposterior $(\mathrm{A})$ and lateral $(\mathrm{B})$ radiographs showed lumbar spondylosis with discovertebral degeneration at $L 4-5$. (C, D) T2-weighted axial magnetic resonance imaging showed previous left partial hemilaminectomy performed at the $L 4$ vertebral lamina. (E, F) It was also observed that the left S1 nerve root was compromised by moderate left subarticular disc extrusion with downward migration. A preganglionic epidural injection was performed through the translateral recess approach. (G) In the anteroposterior fluoroscopic image, the contrast spread pattern for periradicular infiltration appeared as a hummingbird at the L5 exiting (beak, head and wings of a bird) and S1 traversing (abdomen and tail of a bird) nerve roots because of the filling defect caused by subarticular disc extrusion and downward migration. Gray curved line: periradicular infiltration pattern of the $L 5$ exiting nerve root. White curved dotted line: periradicular infiltration pattern of the $S 1$ traversing nerve root. White oval: filling defect of contrast dye for intervertebral disc herniation. (H) Photograph of a real hummingbird for comparison. (I) In the lateral fluoroscopic image, cranial and caudal contrast spread in the ventral epidural space was observed. Gray straight line: dorsal epidural contrast spread. Gray curved line: ventral epidural contrast spread. White dotted line: periradicular infiltration pattern of the L5 exiting nerve root.

Table 1. Anatomical Characteristics of Lower Lumbar Spine

\begin{tabular}{|c|c|c|c|c|c|c|}
\hline Variable & L3 & L4 & L5 & L3-4 & L4-5 & L5-S1 \\
\hline Laminar height (mm) & $22.7 \pm 2.0$ & $20.4 \pm 3.0$ & $16.6 \pm 3.1$ & & & \\
\hline Laminar width (mm) & $13.0 \pm 1.5$ & $14.3 \pm 1.9$ & $15.7 \pm 2.0$ & & & \\
\hline Laminar slop angle $\left({ }^{\circ}\right)$ & $129.0 \pm 7.5$ & $117.5 \pm 4.0$ & $115.7 \pm 5.7$ & & & \\
\hline Laminar overhanging (mm) & $3.0 \pm 2.4$ & $3.4 \pm 2.2$ & $2.2 \pm 2.3$ & & & \\
\hline $\mathrm{DSD}(\mathrm{mm})$ & & & & $14.94 \pm 2.56$ & $15.35 \pm 2.65$ & $19.01 \pm 3.01$ \\
\hline IPD (mm) & & & & $23.25 \pm 2.33$ & $26.45 \pm 2.52$ & $30.59 \pm 2.95$ \\
\hline ILW (mm) & & & & $18.1 \pm 2.9$ & $23.5 \pm 4.7$ & $31.0 \pm 4.7$ \\
\hline DSD/IPD (\%) & & & & 64 & 58 & 62 \\
\hline DSD/ILW (\%) & & & & 82 & 65 & 61 \\
\hline
\end{tabular}

Values are presented as mean \pm standard deviation.

DSD: dural sac anteroposterior diameter, IPD: interpedicular distance, ILW: interlaminar width. 
Hwang et al. Translateral Recess Epidural Injection

Clinics in Orthopedic Surgery • Vol. 11, No. 1, 2019• www.ecios.org

\section{CONFLICT OF INTEREST}

No potential conflict of interest relevant to this article was reported.

\section{REFERENCES}

1. Manchikanti L, Pampati V, Falco FJ, Hirsch JA. Growth of spinal interventional pain management techniques: analysis of utilization trends and Medicare expenditures 2000 to 2008. Spine (Phila Pa 1976). 2013;38(2):157-68.

2. Bicket MC, Horowitz JM, Benzon HT, Cohen SP. Epidural injections in prevention of surgery for spinal pain: systematic review and meta-analysis of randomized controlled trials. Spine J. 2015;15(2):348-62.

3. Kabatas S, Cansever T, Yilmaz C, et al. Transforaminal epidural steroid injection via a preganglionic approach for lumbar spinal stenosis and lumbar discogenic pain with radiculopathy. Neurol India. 2010;58(2):248-52.

4. Kortelainen P, Puranen J, Koivisto E, Lahde S. Symptoms and signs of sciatica and their relation to the localization of the lumbar disc herniation. Spine (Phila Pa 1976). 1985;10(1):88-92.
5. Ebraheim NA, Miller RM, Xu R, Yeasting RA. The location of the intervertebral lumbar disc on the posterior aspect of the spine. Surg Neurol. 1997;48(3):232-6.

6. Shim DM, Choi YH, Yang JH, Oh SK, Kim CG, Park JH. Analysis and measurement of the lumbar spinal canal dimension using magnetic resonance imaging. J Korean Orthop Assoc. 2008;43(5):588-94.

7. $\mathrm{Xu}$ R, Burgar A, Ebraheim NA, Yeasting RA. The quantitative anatomy of the laminas of the spine. Spine (Phila $\mathrm{Pa}$ 1976). 1999;24(2):107-13.

8. Kim ED, Roh MS, Park JJ, Jo D. Comparison of the ventral epidural spreading in modified interlaminar approach and transforaminal approach: a randomized, double-blind study. Pain Med. 2016;17(9):1620-7.

9. Jasper JF. Lumbar retrodiscal transforaminal injection. Pain Physician. 2007;10(3):501-10. 